\title{
Periodic Energy-Optimal Path Planning for Solar-Powered Aircraft
}

\author{
Sara C. Spangelo* Elmer G. Gilbert $\dagger$ Andrew T. Klesh $\ddagger$ \\ Pierre T. Kabamba $\$$ and Anouck R. Girard ${ }^{\uparrow}$ \\ University of Michigan, Ann Arbor, Michigan, 48109
}

\begin{abstract}
[Abstract] This paper considers energy-optimal path planning in a loitering mission for solar-powered unmanned aerial vehicles (UAVs) which collect solar energy from the sun to power their flight. We consider ascending and descending flight maneuvers in a periodic mission constrained to the surface of a vertical cylinder. The coupling of the aircraft kinematic and energetic models is treated in a novel scheme that implements both the periodic and cylindrical constraints. Optimum trajectories are identified by specifying the heading angle and altitude by periodic splines. Given the periodic splines, we are able to solve for the other aircraft parameters, including the aerodynamic, propulsive, and energetic properties of the aircraft. In an example problem, trajectories are obtained that generate better energy properties than those given by constant altitude circular flight. Numerical simulation results are presented that help demonstrate the properties of the optimum trajectories.
\end{abstract}

\section{Nomenclature}

$\begin{array}{llll}\alpha & \text { Angle of attack, deg } & \gamma & \text { Flight path angle, deg } \\ \bar{a} & \text { Acceleration, } m / s^{2} & L & \text { Incidence angle of sun rays, deg } \\ \tilde{a} & \text { Azimuth of the sun, deg } & K & \text { Magnitude of Lift, } \mathrm{N} \\ D & \text { Drag of the aircraft, } \mathrm{N} & P_{s d} & \text { Power spectral density of the sun, } W / m^{2} \\ e & \text { Elevation of the sun, deg } & \psi & \text { Heading, deg } \\ E_{T o t a l} & \text { Total energy, J } & r & \text { Radius of flight cylinder, m } \\ \tau & \text { Period, 1/s } & T & \text { Thrust of the aircraft, } \mathrm{N} \\ E_{R} & \text { Energy Ratio } & \theta & \text { Pitch angle, deg } \\ E_{C P_{R}} & \text { Energy Improvement over Constant Altitude Flight } & \bar{V} & \text { Velocity, m/s } \\ \eta_{\text {prop }} & \text { Efficiency of the propeller } & W & \text { Weight of the aircraft, } \mathrm{N} \\ \eta_{\text {sol }} & \text { Efficiency of the solar cells } & z & \text { Vertical altitude, } \mathrm{m}\end{array}$

\section{Introduction}

This paper is motivated by long endurance missions, such as surveillance of an object of interest for an extended period of time. The missions are executed by unmanned aerial vehicles (UAVs) that are equipped with solar cells on the upper surface of the wings. Solar energy is collected from the sun throughout flight, and the excess energy is stored in an on-board battery. A propeller driven by the energy in the battery is used to sustain flight even when there is little or no available solar energy.

*Graduate Student, Aerospace Engineering, 1320 Beal Ave, Ann Arbor, MI 48109, saracs@umich.edu

${ }^{\dagger}$ Professor Emeritus, Aerospace Engineering, 1320 Beal Ave, Ann Arbor, MI 48109, elmerg@umich.edu@umich.edu

¥Postdoctoral Researcher, Aerospace Engineering, 1320 Beal Ave, Ann Arbor, MI 48109, aklesh@umich.edu

§Professor, Aerospace Engineering, 1320 Beal Ave, Ann Arbor, MI 48109, kabamba@umich.edu

『Assistant Professor, Aerospace Engineering, 1320 Beal Ave, Ann Arbor, MI 48109, anouck@umich.edu 
This paper studies energy-optimal path planning for solar-powered UAVs. The path planning problem features the interaction between three subsystems: aircraft kinematics, energy collection, and energy loss. In the surveillance problem, the UAV must fly periodic paths along the surface of a vertical circular cylinder to survey an object of interest. To satisfy the mission constraints and minimum-energy objectives of the problem, we fly periodic paths along the surface of a vertical cylinder. The flight paths are periodic in the sense that the initial and final aircraft position and configuration are identical. We are motivated to study ascending and descending periodic flight paths because of their potential advantage of the aircraft flying at more favorable wing angles relative to the sun, compared to steady level circular flight. Consider, for example, flight when the sun is at a low elevation. By performing a quick ascent followed by a slow descent, the wing may have superior exposure to the sun, in both ascent and descent, yielding a more energy-efficient flight.

The current literature examines the coupling of energy collection and energy loss with the aircraft kinematics in constant altitude flight, but the effects of pitching and periodic ascending and descending flight maneuvers have not been studied. This paper makes two main contributions.

- A novel spline-based optimization procedure is described that effectively maximizes energy gain subject to the periodic and cylindrical constraints.

- Numerical results show that the optimal trajectories produce improvement in energy properties relative to constant speed and altitude circular flight.

Minimum power consumption in level flight and the geometric configuration of the aircraft have been the major research areas of solar-powered flight. ${ }^{1-28}$ A review of the general history and existing literature on the analysis and design of solar powered flight is provided in Ref. 29. Optimization-based procedures considering flight maneuvers and sunlight availability are introduced to design the aircraft in Ref. 13, 16, and 27. Path planning for solar-powered aircraft generally focuses on mission design, such as Ref. 28,30-32, and the preliminary design of energy efficient flight, Ref. 33-39.

Solar-powered aircraft have potential uses in both exploration and civilian applications, including flight on other planets such as Mars and Venus, and high-altitude wireless communication (See Refs. 32,40-44). Ref. 40 considers optimal path planning maneuvers including takeoff, cruise, and climb, and Ref. 23 compares solar flight on Mars to flight on the Earth.

The coupling of aircraft kinematics and energetics in energy-optimal path planning for perpetual level flight is considered in Ref. 29. Perpetual endurance is the ability of a solar aircraft to collect more energy from the sun than it expends throughout a solar day. Optimal flight paths are predicted for steady level flight, and the requirement for perpetual flight quantified. The optimal endurance problem for solar-powered aircraft considering the effects of altitude variation is investigated in Ref. 29.

Existing research suggests that aircraft endurance may be improved significantly by periodic control, primarily motivated by fuel conservation of subsonic aircraft. ${ }^{45}$ Periodic altitude-varying flight on the surface of a vertical cylinder for optimal fuel efficiency is considered in Ref. 46-47. The unlimited flight endurance of a manned, solar aircraft is proposed in Ref. 48, where the aircraft dynamics, energy management, and propulsion systems are considered in a periodic day-night cycle. However, the study of energy-optimal path planning for solar UAVs has not considered the effects of periodic altitude varying flight maneuvers.

The remainder of the paper is as follows. The general approach is presented in Section III, while Section IV addresses the computational details of maximizing the total energy of the UAV. Section V considers an example vehicle. Optimal trajectories are computed and their properties discussed. Section VI provides some conclusions and discusses future work. Various supporting details are contained in the Appendices.

\section{The General Approach}

\section{A. Aircraft Model and Kinematics}

\section{Trajectory Specification and Aircraft Model}

The aircraft is assumed to fly through still air and is constrained to the surface of a vertical circular cylinder of radius $r$. Turning is coordinated and the altitude is varied by controlling the pitch. The aircraft trajectory is uniquely and fully parameterized as a function of time by the heading $\psi$, and altitude $z$. The state variables are then obtained from the derivatives of $\psi$ and $z$, and used to solve for the lift, drag, thrust, and the total 
energy. This computational procedure is a direct way of imposing periodic flight and avoids solving the differential equations of motion.

In the inertial $(x, y, z)$ Cartesian coordinate system of Figure 1, it follows that

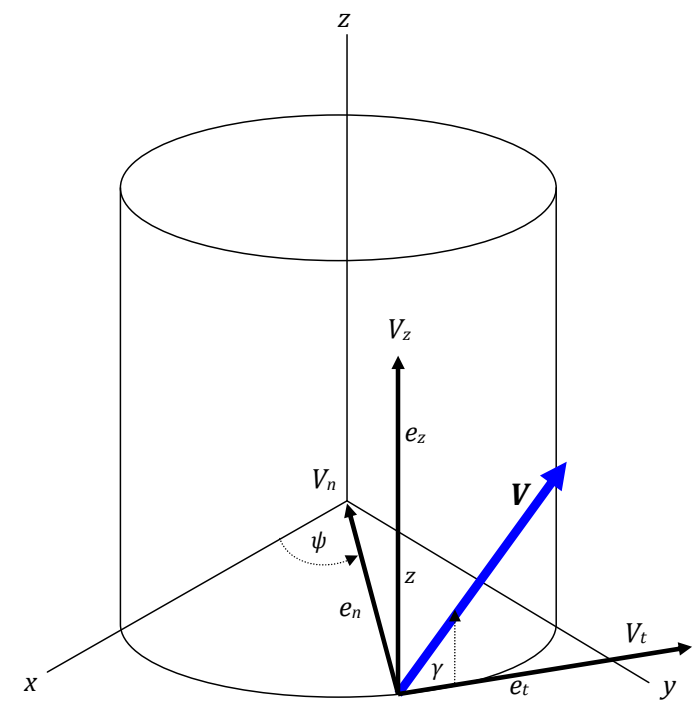

Figure 1. Aircraft Model Coordinate System

$$
\begin{aligned}
& x(t)=r \cos \psi(t), \\
& y(t)=r \sin \psi(t), \\
& z(t)=z(t) .
\end{aligned}
$$

The velocity and the acceleration vectors, $\bar{V}(t)$ and $\bar{a}(t)$, are expressed in terms of a moving coordinate system attached to the aircraft. Let the unit coordinate vectors $e_{t}, e_{n}$, and $e_{z}$ point respectively in directions tangential, normal and vertical to the surface of the cylinder.

Then, the components of $\bar{V}(t)$ and $\bar{a}(t)$ are respectively,

$$
\begin{aligned}
V_{t}(t) & =r \dot{\psi}(t), & a_{t}(t) & =r \ddot{\psi}(t), \\
V_{n}(t) & =0, & a_{n}(t) & =r \dot{\psi}^{2}(t), \\
V_{z}(t) & =\dot{z}(t), & a_{t}(t) & =\ddot{z}(t) .
\end{aligned}
$$

The flight path angle $\gamma(t)$, measured between the aircraft's velocity vector and the unit tangential vector $e_{t}$ in the $e_{t}-e_{z}$ plane, is expressed by

$$
\gamma(t)=\arctan \left(\frac{\dot{z}(t)}{r \dot{\psi}(t)}\right)
$$

\section{Applied Forces and Equations of Motion}

To obtain the equations of motion we introduce the aerodynamic force and thrust vectors, $F^{A}$ and $F^{T}$, expressed in the $e_{t}, e_{n}$, and $e_{z}$ coordinate system. For notational simplicity, the dependence of variables on time, $t$, is not explicitly shown. Zero side-slip is implied through coordinated turning and still air.

The drag part of $F^{A}$ has magnitude $D$ and is aligned with $\bar{V}$ and the lift part, $L^{A}$ is orthogonal to $\bar{V}$. Let $V$ denote the magnitude of $\bar{V}$ and $L$ the magnitude of $L^{A}$, as in Figure 2. The components of $L^{A}$, denoted by $L_{t}, L_{n}, L_{z}$, satisfy the orthogonality condition, 


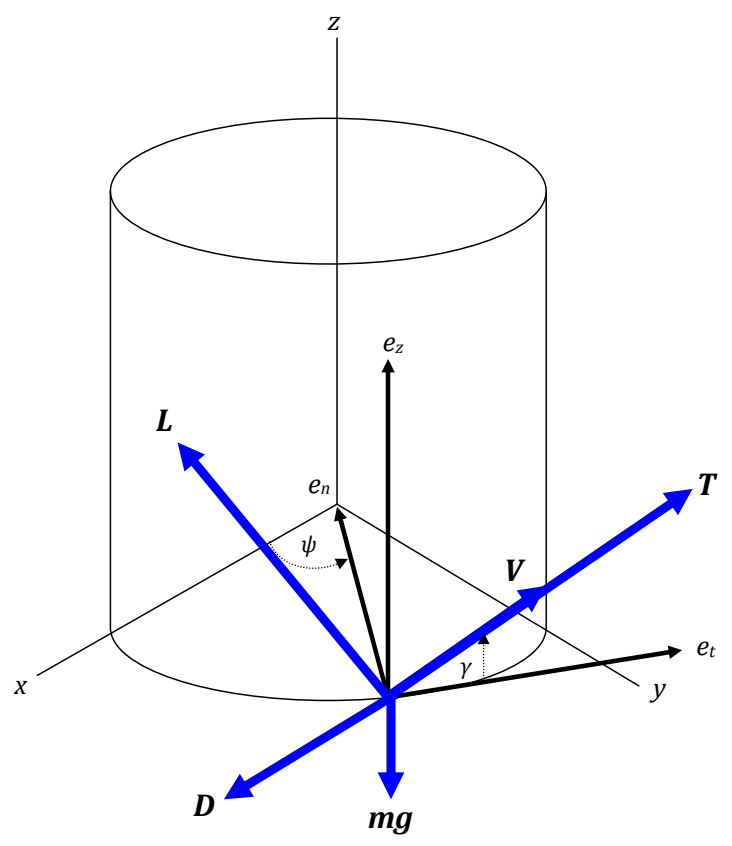

Figure 2. Aircraft Forces

$$
\left(L_{t}\right)(r \dot{\psi})+\left(L_{n}\right)(0)+\left(L_{z}\right)(\dot{z})=0
$$

Then $L_{t}=-\left(\frac{\dot{z}}{r \dot{\psi}}\right) L_{z}$, and there is no constraint on $L_{n}$.

Finally, it is assumed the thrust vector is aligned with $\bar{V}$, which is a reasonable approximation in most cases for small angle of attack, $\alpha<<1^{\circ}$. The angle of attack is the angle between the aircraft body fixed axis perpendicular to the wings and the direction of $\bar{V}$. Let $T$ be the thrust magnitude. Assembling these relations yields the equations of motion:

$$
\begin{aligned}
m r \ddot{\psi} & =(T-D) \cos \gamma+L_{t}, \\
m r \dot{\psi}^{2} & =L_{n}, \\
m \ddot{z} & =-m g+(T-D) \sin \gamma+L_{z},
\end{aligned}
$$

where the drag and the thrust forces are measured along the velocity vector. This assumption on thrust direction is a reasonable approximation since, in general, $T \sin \alpha<<L$.

\section{Aerodynamic Model}

Drag is expressed in the usual way, ${ }^{49}$ 


$$
\begin{aligned}
& D=\frac{1}{2} \rho V^{2} S C_{D}(\alpha), \\
& C_{D}(\alpha)=C_{D o}+K C_{l}^{2}(\alpha), \\
& C_{l}(\alpha)=\frac{2 L}{\rho V^{2} S}=C_{l_{o}}+C_{l_{\alpha}} \alpha,
\end{aligned}
$$

where $\rho$ is the atmospheric density, and $S$ the wing area. $C_{L}(\alpha), C_{L_{o}}$, and $C_{l_{\alpha}}$, and $C_{D o}$ are the lift and drag coefficients parameters. The aerodynamic coefficient $K=\frac{1}{\epsilon \pi A R}$ represents the induced drag exceeding the drag of an elliptical lift distribution. $A R=b^{2} / S$ is the aspect ratio, $b$ is the wing span, and $\epsilon$ is the Oswald efficiency factor. By combining (12)-(14), we solve for $D$,

$$
D=\frac{1}{2} \rho V^{2} S\left[C_{D o}+K\left(\frac{2 L}{S \rho V^{2}}\right)^{2}\right] .
$$

\section{Parameterization of Lift, Drag, and Thrust by $\psi$ and $z$}

It is assumed that $\psi$ and altitude $z$ together with their derivatives $\dot{\psi}, \ddot{\psi}, \dot{z}$, and $\ddot{z}$, are continuous, and, for computing, they are defined by periodic spline functions. The algorithmic steps to solve for $L, D$, and $T$, and other aerodynamic properties using Equations (4)-(15) are outlined in Appendix A.

\section{B. Energy Model}

\section{Energy in Flight}

A standard propulsion model is used to derive the power expended by the aircraft during flight. In powered flight, the propeller losses are a function of thrust $T$, velocity $V$, and the propeller efficiency $\eta_{\text {prop }}$. The propeller is motionless, $T=0$, in gliding flight. Due to the periodic nature of the flight considered, where the aircraft begins and completes one cycle at the same altitude and speed, the kinetic and potential energy is conserved and excluded from the energy modeling. The output power from the energy storage battery is required to sustain flight through the night.

$$
\begin{aligned}
& P_{\text {out }}=\frac{T V}{\eta_{\text {prop }}} \quad \text { if } T \geq 0, \\
& P_{\text {out }}=0 \quad \text { if } T<0 .
\end{aligned}
$$

Negative thrust, if needed, may be generated by the additional operation of flaps or other aerodynamic control surfaces.

The total energy $E_{\text {out }}$ required from the battery over a time interval $\left[t_{o}, t_{f}\right]$ is,

$$
E_{\text {out }}=\int_{t_{o}}^{t_{f}} P_{\text {out }}(t) d t
$$

\section{Energy Collection}

The solar cells are mounted on the top surface of the aircraft wings, such that power is collected from solar rays. The incidence angle, $\kappa$, is the angle between the line-of-sight from the aircraft to the sun and the vertical ascending axis of the aircraft fixed frame. As a function of the sun's elevation $e$ and azimuth $\tilde{a}$ and the aircraft's heading angle $\psi$ and pitch angle $\theta=\gamma+\alpha, \kappa$ is expressed as:

$$
\cos \kappa=-\cos e \sin \theta \sin (\tilde{a}-\psi)+\sin e \cos \theta,
$$

which is derived in Ref. ${ }^{29}$ The solar position model, which governs the sun elevation $e$ and azimuth $\tilde{a}$ is also derived in Ref. ${ }^{29}$ 
The power collected by the aircraft is a function of the solar cell efficiency $\eta_{\text {sol }}$, the solar spectral density $P_{s d}$, the total wing area $S$, and the solar incidence angle $\kappa$ :

$$
P_{i n}=\eta_{s o l} P_{s d} S \cos \kappa
$$

$P_{s}$ is naturally zero for solar incidence angles $\kappa<0$. The solar cell efficiency takes into account a wing that is not entirely covered with solar cells.

The total collected energy, $E_{i n}$,

$$
E_{i n}=\int_{t_{o}}^{t_{f}} P_{i n}(t) d t
$$

\section{Total Energy}

The total energy due to solar collection and the propeller losses over a time interval $\left[t_{o}, t_{f}\right]$ is,

$$
E_{\text {Total }}=E_{\text {in }}-E_{\text {out }}
$$

\section{Summary}

The heading angle and vertical altitude, and their derivatives, govern the variables defined in Equations (4)(7). The expressions for the equations of motion, (8)-(15), affect the power loss, Eqs. (16)-(17). The power collected from the sun, Equation (21), is directly related to the solar incidence angle, Eq. (19), which is a function of the sun's relative position and the heading and pitch angles of the aircraft. Equations (18), (22), and (23) complete the energy model. Under a zero-pitch condition, the kinematic and energetic expressions reduce to the Klesh model. ${ }^{29}$

\section{Computational Maximization of Total Energy}

The optimization problem is to choose $z(t)$ and $\psi(t)$ so that they are smooth periodic functions such that (1)-(22) are satisfied and $E_{\text {Total }}$ is maximized. The computational approach to maximizing $E_{\text {Total }}$ is to specify $z(t)$ and $\psi(t)$ by periodic cubic splines with continuous first and second derivatives. In particular, to maintain spline smoothness, they must satisfy the conditions:

$$
\begin{array}{ll}
z\left(t_{o}\right)=z\left(t_{f}\right)=z_{o}, & \psi\left(t_{o}\right)=\psi\left(t_{f}\right)=\psi_{o}, \\
\dot{z}\left(t_{o}\right)=\dot{z}\left(t_{f}\right)=\dot{z}_{o}, & \dot{\psi}\left(t_{o}\right)=\dot{\psi}\left(t_{f}\right)=\dot{\psi}_{o}, \\
\ddot{z}\left(t_{o}\right)=\ddot{z}\left(t_{f}\right)=\ddot{z}, & \ddot{\psi}\left(t_{o}\right)=\ddot{\psi}\left(t_{f}\right)=\ddot{\psi}_{o},
\end{array}
$$

and pass through specified values at the knot values of $t, t=t_{o}, t_{i}, . . t_{n-1}$, and $t_{n}=t_{f}$. Computational procedures for computing such periodic splines in terms of their knot values are available in Appendix C. When the knot values are specified, it is possible, following the algorithm as described in Appendix A and the general description in Section C, to compute by numerical integration $E_{\text {Total }}$. Having done this, $E_{\text {Total }}$ is then maximized by applying an optimization routine to the knot parameters and the period $\tau=\left(t_{f}, t_{o}\right)$.

While it is possible to compute $E_{\text {Total }}$ when the sun position changes and air density varies with $z$, the position and density are assumed to be constant in the computations that follow. These are likely to be good approximations in most practical applications of short duration missions.

Inequality constraints may also be needed in the optimization of $E_{\text {Total }}$. For example, the angle of attack and altitude are limited to specified intervals in the example in the next Section. Such constraints are difficult to handle and need further attention. The optimization program described in Appendix $\mathrm{C}$ used in the maximization of $E_{\text {Total }}$ implements these reasonably well.

In the computation of the paths in this paper, it is assumed that the period $\tau=t_{f}-t_{o}$ is relatively short so that the average position over the period $\left(t_{f}, t_{o}\right)$ can be approximated as constant. It is also possible to let the sun position change in time, motivating the various sun elevations studied in Section D. 


\section{Results for an Example Aircraft}

This section considers periodic flight paths for an example UAV, the YellowTail described in Appendix $\mathrm{D}$ for its key parameters. The cylinder radius is $r=300 \mathrm{~m}$ and an upper altitude constraint of $H=50 \mathrm{~m}$ is imposed. Optimal trajectories are obtained and then their properties discussed, with emphasis on two non dimensional energy ratios that characterize trajectory performance.

\section{A. Fixed Altitude Circular Trajectories}

As a point of reference, we consider first the energy properties of constant speed and altitude circular flight. The total energy for one cycle of circular flight is obtained from Equations (8)-(23) with $\dot{\psi}=$ constant, and $z=0$. Let the total energy be denoted by $E_{C P T o t a l}$, which clearly depends on the speed $V$ and circular radius $r$. A plot of $E_{C P T o t a l}$ as a function of $V$ and elevation angle is shown in Figure 3. As expected, larger velocities require higher thrust and the resultant increase in propeller power decreases the total energy. The maximum angle of attack stall constraint $\left(\alpha_{\max }=18^{\circ}\right)$ prevents $E_{C P T o t a l}$ from being defined for $V$ less than about $5.5 \mathrm{~m} / \mathrm{s}$.

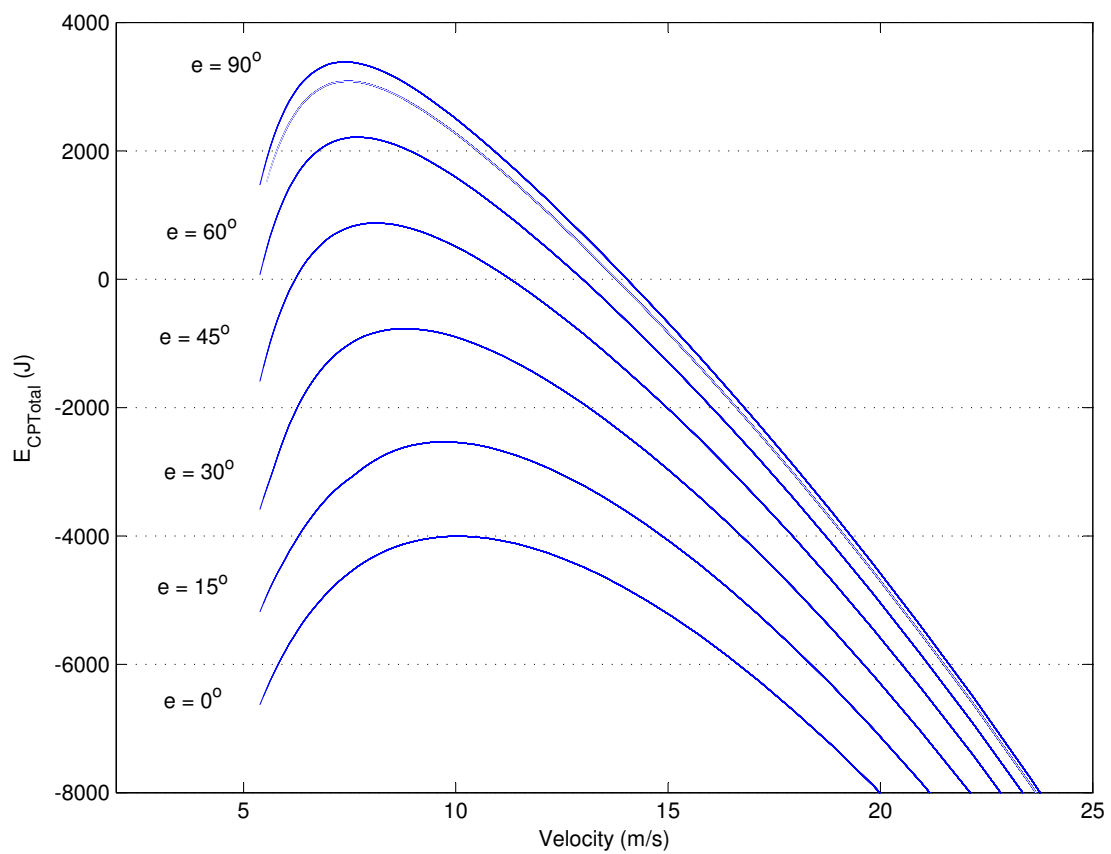

Figure 3. Velocity and Total Energy Results for Constant Altitude Circular Flight

For $e=45^{\circ}$, the optimum speed, period and energy are $V_{C P}=8.43 \mathrm{~m} / \mathrm{s}, \tau_{C P}=230 \mathrm{~s}, E_{C P \text { Total }}=874 \mathrm{~J}$. It can be shown that a very good approximation for the optimum velocity in constant altitude circular flight at large radii is the minimum power velocity, ${ }^{2950}$

$$
V_{\text {Power }_{\text {min }}}=\sqrt[4]{\frac{4 K W^{2}}{3 C_{D_{o}} \rho^{2} S^{2}}}
$$

\section{B. Measuring Flight Performance}

Two non-dimensional energy ratios are useful in evaluating the performance of the periodic flight paths for solar powered aircraft. 
In practical flight applications, we are interested in maximizing total energy after one full period. The energy ratio

$$
E_{C P_{R}}=\frac{E_{\text {Total }}-E_{C P T o t a l}}{E_{C P T o t a l}} \cdot 100 \% .
$$

measures fractional improvements of the $E_{\text {Total }}$ relative to $E_{C P T o t a l}$. When $E_{C P_{R}}>1$, the trajectory corresponding to $E_{\text {Total }}$ generates more energy than the circular fixed altitude path.

Let

$$
E_{R}=\frac{E_{\text {in }}}{E_{\text {out }}}=\frac{\int_{t_{o}}^{t_{f}} P_{\text {in }}(t) d t}{\int_{t_{o}}^{t_{f}} P_{\text {out }}(t) d t} .
$$

This energy ratio compares the relative amount of energy collected and the amount of energy expended in flight.

When $E_{R}>1$, the trajectory corresponding to $E_{\text {Total }}$ generates an increase in the energy stored in the battery. Unlike $E_{C P_{R}}, E_{R}$ does not require knowledge of the optimal fixed altitude circular path.

In steady level straight flight, $E_{R}$ equals the power ratio, ${ }^{29} P_{R}=\frac{P_{i n}}{P_{\text {out }}}$. However, this result is not valid for altitude dependent trajectories on the surface of a cylinder.

An alternative to maximizing $E_{\text {Total }}$ is to maximizing $E_{R}$, the energy results are extremely close.

\section{Optimal Periodic Flight Paths}

1. Example Flight Path

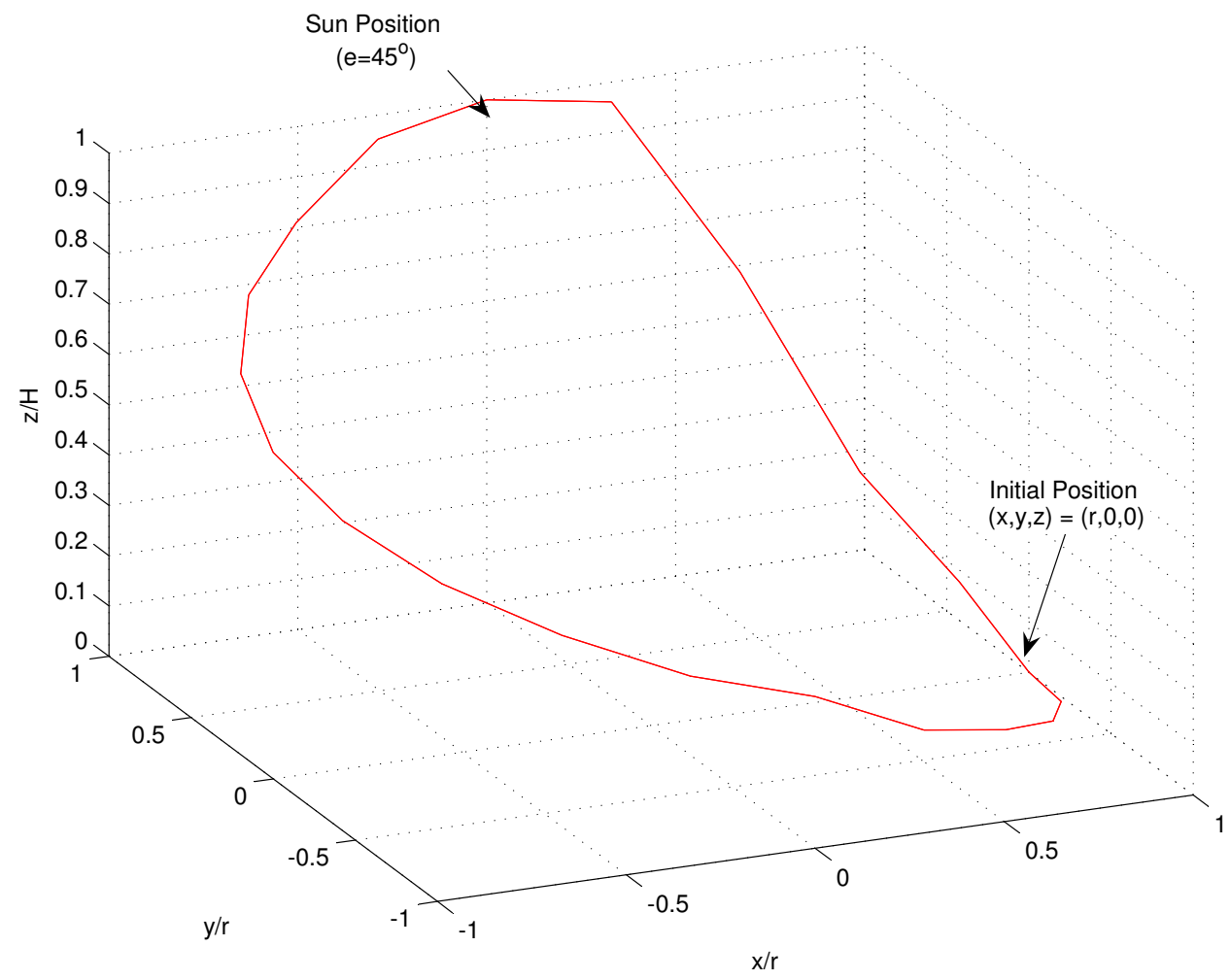

Figure 4. Representative Flight Path, $e=45^{\circ}, r=300 m, H=50 m$ 
a) Vertical Altitude

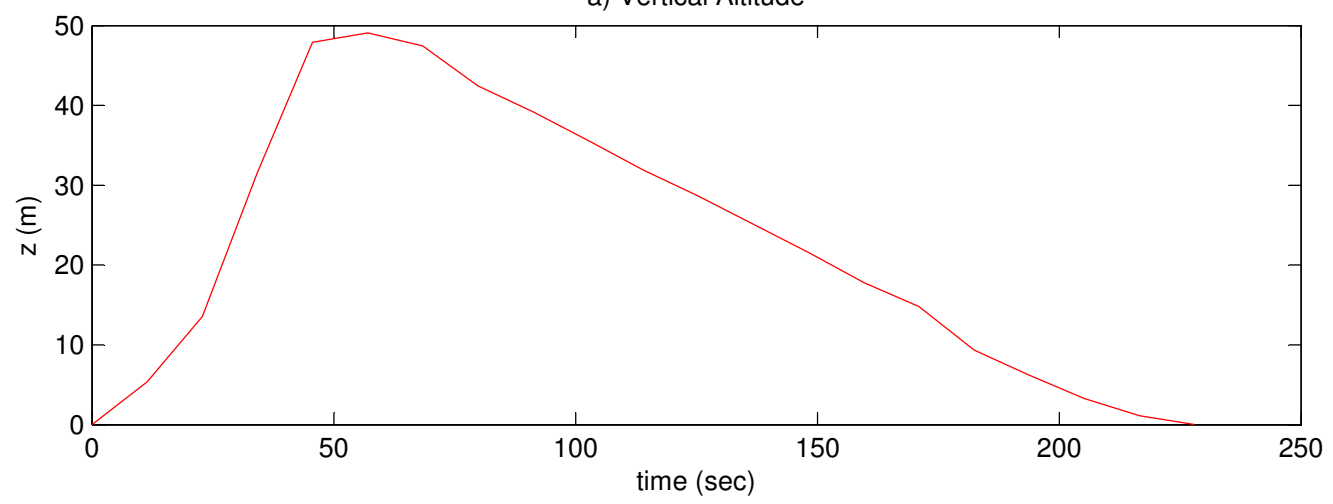

b) Descent and Ascent Rates

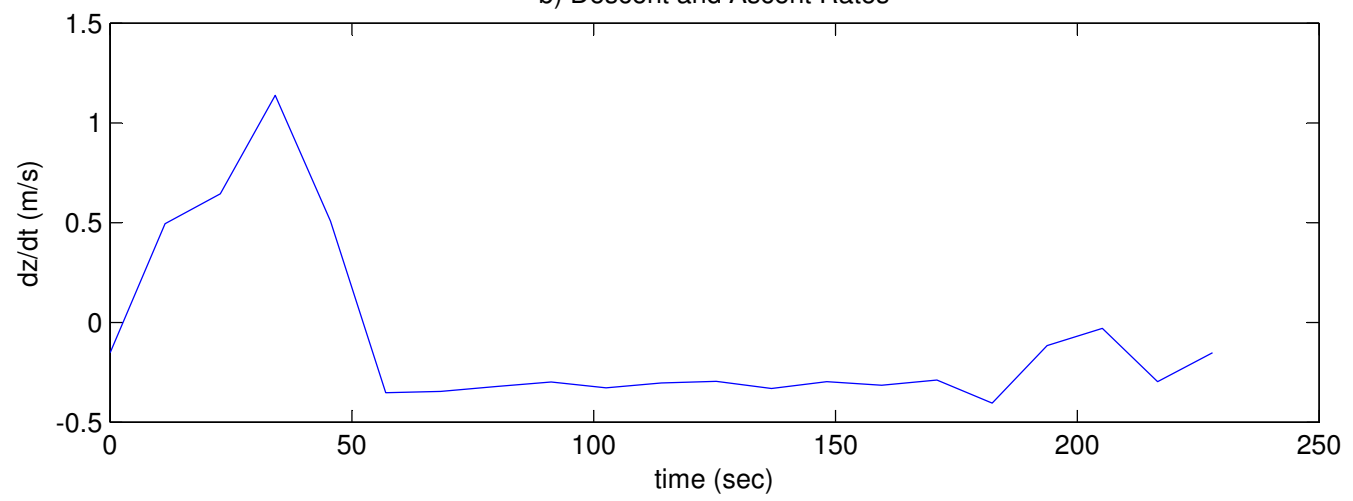

Figure 5. Spline for Altitude $z$ and Derivative $\dot{z}$

An example energy-optimal periodic flight path is shown in Figure 4. The optimal flight period and trajectories are obtained as discussed in Section, where $z(t)$ is represented by a periodic spline function and $\dot{\psi}(t)=\frac{2 \pi t}{\tau}$. The linear dependence of $\psi(t)$ is probably close to optimum and it eliminates the requirement for a spline representation of $\psi(t)$.

Figure 6 shows how the remaining aircraft variables depend on $t$. The flight path angle and velocity increase throughout the initial climb, requiring high thrust and power values to gain altitude rapidly. The low $\gamma$ descent maintains an energy-efficient speed; thereby reducing thrust and power expenditure. The improvement for this flight path as compared to steady level flight is $E_{C P_{R}}=9.58 \%$. We recognize small oscillations exist in the spline function, indicating numerical condition problems that need attention.

\section{Solar Effects in Energy-Optimal Path Planning}

\begin{tabular}{|c|c|c|}
\hline Elevation $(e)$ & $E_{R}$ & $E_{C P_{R}}$ \\
\hline $15^{\circ}$ & 1.68 & $162.37 \%$ \\
\hline $30^{\circ}$ & 3.32 & $18.60 \%$ \\
\hline $45^{\circ}$ & 4.74 & $9.58 \%$ \\
\hline $60^{\circ}$ & 5.83 & $6.75 \%$ \\
\hline $75^{\circ}$ & 6.53 & $5.77 \%$ \\
\hline $90^{\circ}$ & 6.79 & $5.77 \%$ \\
\hline
\end{tabular}

Table 1. Effects of Elevation on Energy Properties 

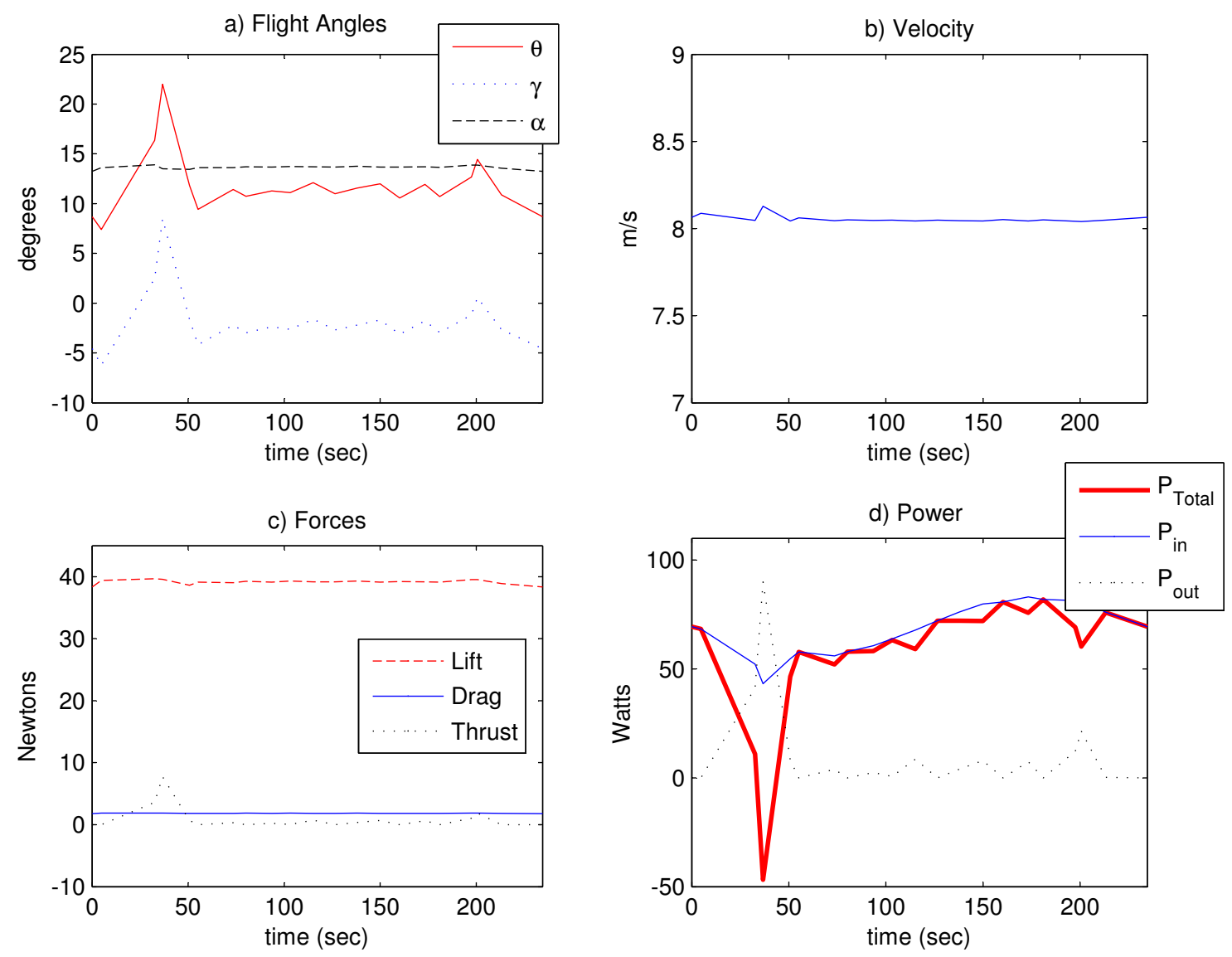

Figure 6. Time History of Flight Parameters

The $E_{C P_{R}}$ for different solar elevations is shown in Figure 7. When the sun is low in the sky, there is no gain in flying periodic altitude dependent flight paths. Pitching the aircraft to climb and glide does not improve the angles of the wing relative to the sun sufficiently to compensate for the additional propeller losses. The greatest improvement are realized when $e=15^{\circ}$, and with increasing solar elevation the relative improvement by periodic altitude dependent paths decreases. It is interesting to note that $E_{C P_{R}}>1$ even when the sun is directly overhead $\left(e=90^{\circ}\right)$. This surprising result shows that the optimization routine has taken advantage of the coupling between energy-efficient climbs and efficient glides (See Ref. 45). Simulation results for different solar azimuth angles yield identical results due to the periodic nature of the paths. Table D shows the energy ratio and $E_{C P_{R}}$ as a function of sun elevation $e$.

\section{Conclusions}

We have developed an approach for optimizing for the periodic motion of a solar powered UAV in circular, altitude-dependent loitering. It exploits reasonable aircraft and energy collection models. The periodic, spline-based computational procedure is an effective way of imposing the usually difficult to impose periodic boundary conditions. We evaluate the performance of these paths by the two non-dimensional energy ratios $E_{C P_{R}}$ and $E_{R}$. The positive results shown in Figure 7 are at least a proof in principle of altitude dependent loitering has an energy advantage.

Future work will investigate further computational aspects of the optimization problem including the search for more complex trajectories such as those with several revolutions of the gliding descent. The effects of variation in altitude and wind will also be considered. 


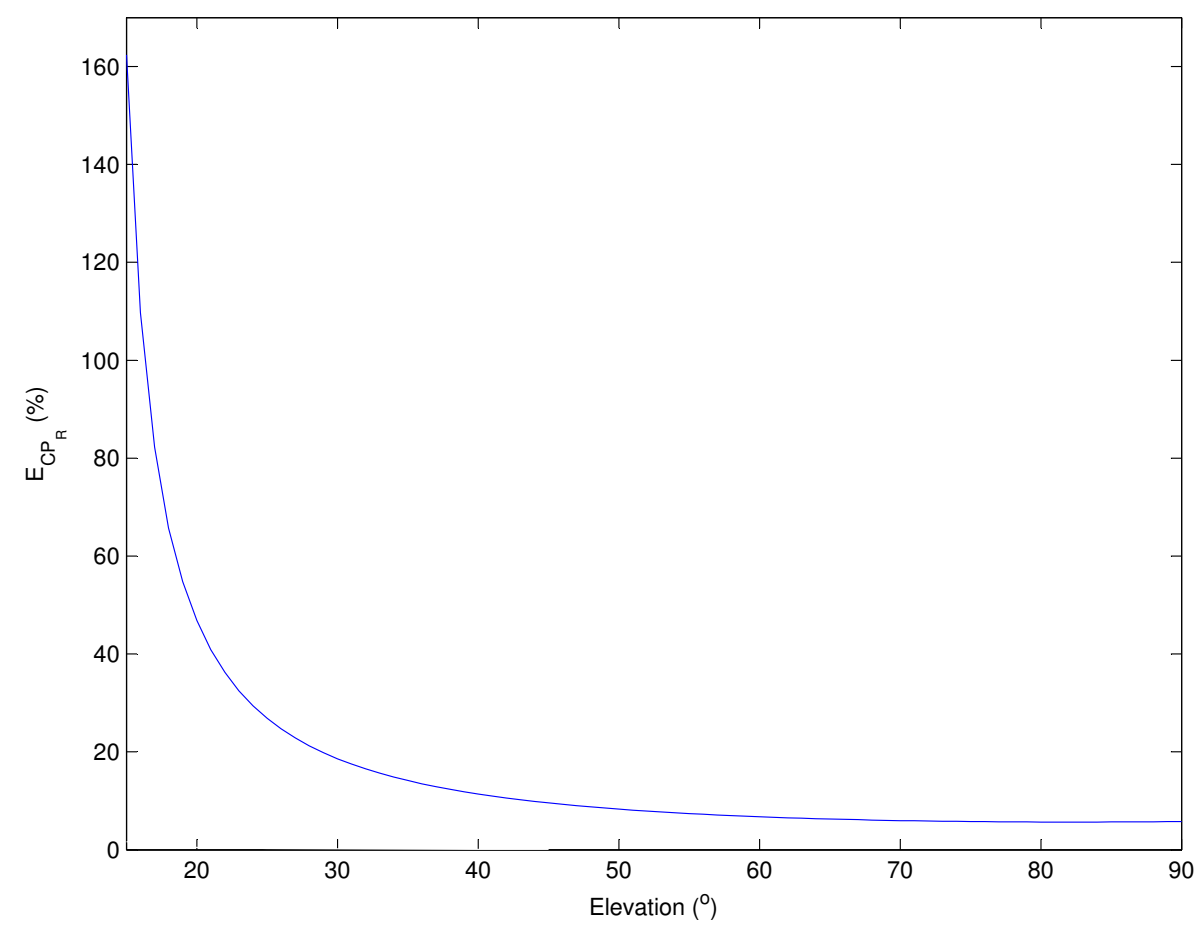

Figure 7. Improvement in Total Energy $E_{C P_{R}}$ in Periodic Flight for Variable Sun Elevations, $\tilde{a}=-90^{\circ}, r=300 m$ 


\section{References}

${ }^{1}$ F. G. Irving and D. Morgan, "The feasibility of an aircraft propelled by solar energy," in 2nd International Symposium on the Technology and Science of Low Speed and Motorless Flight, AIAA, Sep 1974.

${ }^{2}$ D. W. Hall, C. D. Fortenbach, E. V. Dimiceli, and R. W. Parks, "A Preliminary Study of Solar Powered Aircraft and Associated Power Trains," NASA Contractor Report 3699, Dec 1983.

${ }^{3}$ R. J. Boucher, "History of Solar Flight," in 20th Joint Propulsion Conference, SAE and ASME, Jun 1984.

${ }^{4}$ N. J. Colella and G. S. Wenneker, "Pathfinder and the Development of Solar Rechargable Aircraft," Energy and Technology Review, pp. 1-9, 1994.

${ }^{5}$ N. Baldock and M. R. Mokhtarzadeh-Dehghan, "A study of solar-powered, high-altitude unmanned aerial vehicles," Aircraft Engineering and Aerospace Technology, vol. 78, no. 3, pp. 187-193, 2006.

${ }^{6}$ William H. Phillips, "Some Design Considerations for Solar-Powered Aircraft," NASA Technical Report 1675, Jun 1980.

${ }^{7}$ P. B. MacCready, P. B. S. Lissaman, W. R. Morgan, and J. D. Burke, "Sun Powered Aircraft Design," in Annual Meeting and Technical Display on Frontiers of Achievement, AIAA, May 1981.

${ }^{8} \mathrm{~J} . \mathrm{W}$. Youngblood and T. A. Talay, "Solar-powered airplane design for long-endurance, high altitude flight," in $2 n d$ International Verh Large Vehicles Conference, AIAA, May 1982.

${ }^{9} \mathrm{~J}$. W. Youngblood, T. A. Talay, and R. J. Pegg, "Design of long-endurance unmanned airplanes incorporating solar and fuel cell propulsion," in 20th Joing Propulsion Conference, SAE and ASME, Jun 1984.

${ }^{10}$ P. M. Stella and D. J. Flood, "Photovoltaic Options for Solar Electric Propulsion," NASA Technical Memorandum 103284, Jul 1990.

${ }^{11}$ S. A. Brandt and F. T. Gilliam, "Design Analysis Methodology for Solar-Powered Aircraft," Journal of Aircraft, vol. 32, no. 4, pp. 703-709, 1995.

${ }^{12}$ K. C. Reinhardt, T. R. Lamp, J. W. Geis, and A. J. Colozza, "Solar-powered unmanned aerial vehicles," in 31st Intersociety Energy Conversion Engineering Conference, IECEC, Aug 1996.

${ }^{13}$ O. Trifu and G. Savu, "Unmanned solar powered aerial surveyor configured with an aerodynamic optimization procedure," in 15th AIAA Applied Aerodynamics Conference, AIAA, Jun 1997.

${ }^{14}$ A. J. Colozza, D. A. Scheiman, and D. J. Brinker, "GaAs/Ge Solar Powered Aircraft," NASA Technical Memorandum 1998-208652, Oct 1998.

${ }^{15}$ K. Flittie and B. Curtin, "Pathfinder solar-powered aircraft flight performance," in Atmospheric Flight Mechanics Conference and Exhibit, AIAA, Aug 1998.

${ }^{16} \mathrm{P}$. Berry, "The Sunriser - A design study in solar powered flight," in 2000 World Aviation Conference, AIAA and SAE, Oct 2000 .

${ }^{17}$ C. Wilson, J. Nutbean, and I. Bond, "Aerodynamic and Structural Design of a Solar-Powered Micro Unmanned Air Vehicle," Proceedings of the Institution of Mechanical Engineers, Part G: Journal of Aerospace Engineering, vol. 214, no. 2, pp. 97-106, 2000.

${ }^{18}$ G. Frulla, "Preliminary reliability design of a solar-powered high-altitude very long endurance unmanned air vehicle," Proceedings of the Institution of Mechanical Engineers, Part G: Journal of Aerospace Engineering, vol. 216, no. 4, pp. 189-196, 2002.

${ }^{19}$ C. K. Patel, H. Arya, and K. Sudhakar, "Design, Build and Fly a Solar Powered Aircraft," in International Seminar and Annual General Meeting of the Aeronautical Society of India, Aeronautical Society of India, 2002.

${ }^{20}$ C. Roberts, M. Vaughan, and W. J. Bowman, "Development of a Solar Powered Micro Air Vehicle," in 40th Aerospace Sciences Meeting and Exhibit, AIAA, Jan 2002.

${ }^{21}$ M. A. Dornheim, "Get me through the night," Aviation Week and Space Technology, vol. 159, pp. 66, 68, 70, Sep 2003.

${ }^{22}$ A. Colozza, "Solid State Aircraft," Phase I Final Report, prepared for NASA Institute for Advanced Concepts, Nov, 2002.

${ }^{23}$ A. Noth, W. Engel, and R. Siegwart, "Design of an Ultra-Lightweight Autonomous Solar Airplane for Continuous Flight," Proceedings of Field and Service Robotics, Port Douglas, Australia, 2005.

${ }^{24}$ C. R. Theodore, M. B. Tischler, and J. D. Colbourne, "Rapid Frequency-Domain Modeling Methods for Unmanned Aerial Vehicle Flight Control Applications," Journal of Aircraft, vol. 41, pp. 735-743, Aug 2004.

${ }^{25}$ M. A. Dornheim, "SoLong Solar-Powered Drone Stays Aloft for 48 Hr.," Aviation Week and Space Technology, Jun 2005.

${ }^{26}$ G. Romeo, G. Frulla, E. Cestino, and F. Borello, "SHAMPO: Solar HALE Aircraft for Multi Payload and Operations," Aerotecnica Missili e Spazio, vol. 85, Sep 2005.

${ }^{27}$ G. Romeo and G. Frulla, "HELIPLAT: Aerodynamic and Structural Analysis of Solar Powered Platform," in 1st Technical Conference and Workshop on Unmanned Aerospace Vehicles, AIAA, May 2002.

${ }^{28}$ M. A. Dornheim, "Solar Powered Research and Dryden," Webpage, Available at: http://www.nasa.gov/centers/dryden/news/FactSheets/FS-054-DFRC.html, 2006.

${ }^{29}$ A. T. Klesh and P. T. Kabamba, "Solar-Powered Aircraft: Energy-Optimal Path Planning and Perpetual Endurance." Submitted, 2008.

${ }^{30}$ A. J. Colozza, "Effect of Date and Location on Maximum Achievable Altitude for a Solar Powered Aircraft," NASA Contractor Report 202326, Mar 1997.

${ }^{31}$ D. W. Hall, D. A. Watson, R. P. Tuttle, and S. A. Hall, "Mission analysis of solar powered aircraft," NASA Contractor Report 172583, Jul 1985.

${ }^{32}$ A. J. Colozza, "Effect of Power System Technology and Mission Requirements on High Altitude Long Endurance Aircraft," NASA Contractor Report 194455, Feb 1994.

${ }^{33}$ D. F. Chichka and J. L. Speyer, "Solar-powered, formation-enhanced aerial vehicle systems for sustained endurance," in 1998 American Control Conference, IEEE, Jun 1998. 
${ }^{34}$ A. J. Colozza, "Convective Array Cooling for a Solar Powered Aircraft," NASA Contractor Report 212084, Jan 2003.

${ }^{35}$ M. J. Allen, "Updraft model for development of autonomous soaring uninhabited air vehicles," in Proceedings of the ICAE 2003 Conference, ICAE, 1996.

${ }^{36}$ M. J. Allen, "Autonomous soaring for improved endurance of a small uninhabited air vehicle," in Proceedings of the 43rd Aerospace Sciences Meeting, AIAA, 2005.

${ }^{37}$ M. J. Allen, "Guidance and control of an autonomous soaring uav," NASA Technical Memorandum 214611, February 2007.

${ }^{38}$ Y. Qi and Y. Zhao, "Energy-efficient trajectories of unmanned aerial vehicles flying through thermals," Journal of Aerospace Engineering, pp. 84-92, April 1996.

${ }^{39}$ D. E. Metzger and J. K. Hedrick, "Optimal flight paths for soaring flight," in Proceedings of the 2nd International Symposium on the Technology and Science of Low Speed and Motorless Flight, AIAA, 1974.

${ }^{40}$ E. H. Teets Jr, C. J. Donohue, and P. T. Wright, "Meterological Support of the Helios World Record High Altitude Flight to 96,863 Feet," NASA Technical Memorandum 210727, May 2002.

${ }^{41}$ J. F. Gundlach IV, "Umanned solar-powerd hybrid airships for Mars exploration," in 37th Aerospace Sciences Meeting and Exhibit, AIAA, Jan 1999.

${ }^{42}$ S. C. Smith, A. S. Hahn, W. R. Johnson, D. J. Kinney, J. A. Politt, and J. J. Reuther, "The design of the Canyon Flyer, an airplane for Mars exploration," in 38th Aerospace Sciences Meeting and Exhibit, AIAA, Jan 2000.

${ }^{43}$ A. J. Colozza, "Solar Powerd Flight on Venus," NASA Contractor Report 213052, Apr 2004.

${ }^{44}$ A. Noth, W. Engel, and R. Siegwart, "Flying Solo and Solar to Mars - Global Design of a Solar Autonomous Airplane for Sustainable Flight," IEEE Robotics and Automation Magazine, vol. 13, pp. 44-52, Sep 2006.

${ }^{45}$ D. T. Lyons, "Improved Aircraft Cruise by Periodic Control," Ph.D. Dissertation, Department of Aerospace Engineering, The University of Michigan, 1980.

${ }^{46}$ J. L. S. R. H. Chen, "Improved endurance of optimal periodic flight," Journal of Guidance, Control and Dynamics, vol. 30, no. 4, pp. 11231133, year $=2007$.

${ }^{47}$ J. L. S. R. H. Chen, "Improved endurance of optimal periodic flight," in AIAA Guid- ance, Navigation, and Control Conference and Exhibit, AIAA, Aug 2006.

${ }^{48}$ J. L. G. Sachs1 and F. Holzapfel, "Periodic optimal control for solar aircraft with unlimited endurance capability," in AIAA Atmospheric Flight Mechanics Conference and Exhibit, AIAA, Aug 2008.

${ }^{49}$ D. E. B. Thomas R. Yechout, Steven L. Morris and W. F. Hallgren, Introduction to aircraft flight mechanics. AIAA, illustrated ed., 2003.

${ }^{50}$ J. D. Anderson, Aircraft Performance and Design. McGraw-Hill, international edition ed., Jun 1999. 


\section{A. Algorithm for Solving for L, T, D, and $\alpha$}

The following algorithm is based on Eqs. (7)-(11). Given $\psi(t)$ ad $z(t)$ and their derivatives, it determines $L(t), T(t), a d D(t)$ and $\alpha(t)$. For simplicity the argument $t$ is not shown.

1. Set $\gamma=\arctan \left(\frac{\dot{\psi} r}{\dot{z}}\right)$ (Eq. (7)),

2. Set $V=\sqrt{(\dot{z})^{2}+(r \dot{\psi})^{2}}$,

3. Set $L_{n}=m r \dot{\psi}^{2}$ (Eq. (10)),

4. Set

$$
L_{z}=\frac{m \ddot{z}+m g-m r \ddot{\psi} \tan (\gamma)}{\left(\frac{\dot{z}}{r \dot{\psi}}\right) \tan (\gamma)+1}
$$

This result is obtained by multiplying Eq. (9) by $\tan (\gamma)$ and subtraction of Eq. (11).

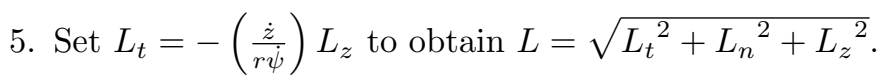

6. Find drag from Eq. (15),

7. Obtain $T$ from Eq. (9),

8. Obtain $\alpha$ from Eq. 14.

\section{B. Incidence Angle}

The incidence angle, $\kappa$, is the angle between the line-of-sight from the aircraft to the sun and the vertical ascending axis of the aircraft fixed frame and is derived as follows.

Let $[g]=\left[\hat{x}_{g}, \hat{y}_{g}, \hat{z}_{g}\right]^{T}$ be the matrix of unit vectors, directed respectively north, east, and vertical ascending. Then,

$$
\hat{s}=[g]^{T}\left[\begin{array}{c}
\cos (e) \cos (\tilde{a}) \\
\cos (e) \sin (\tilde{a}) \\
\sin (e)
\end{array}\right] .
$$

Let $[v]=\left[\hat{x}_{v}, \hat{y}_{v}, \hat{z}_{v}\right]^{T}$ be the matrix of unit vectors, where $\hat{x}_{v}$ and $\hat{y}_{v}$ form the plane along the top surface of the aircraft, and $\hat{z}_{v}$ is parallel to the lift vector. In terms of $[g]$,

$$
[v]=R_{2}(\theta) R_{3}(\psi)[g],
$$

where the pitch angle is $\theta=\gamma+\alpha$ and the rotation matrices are defined,

$$
\begin{gathered}
R_{2}(\theta)=\left[\begin{array}{ccc}
\cos (\theta) & 0 & \sin (\theta) \\
0 & 1 & 0 \\
-\sin (\theta) & 0 & \cos (\theta)
\end{array}\right], \\
R_{3}(\psi)=\left[\begin{array}{ccc}
\cos (\psi) & \sin (\psi) & 0 \\
-\sin (\psi) & \cos (\psi) & 0 \\
0 & 0 & 1
\end{array}\right],
\end{gathered}
$$


represent rotation matrices about the second third axis, respectively. By inverting this relationship, we obtain

$$
[g]=R_{3}^{T}(\psi) R_{2}^{T}(\theta)[v]
$$

The incidence angle $\kappa$ as the angle between the line-of-sight to the sun and the $\hat{z}$-axis of the aircraft-fixed vectrix, then $\kappa=\arccos \left(\hat{s} \cdot \hat{z}_{v}\right)$. Assuming a zero dihedral wing configuration angle, the incidence angle of the sun rays on the solar cells is expressed from Eq. (31) as:

$$
\cos (\kappa)=-\cos (e) \sin (\theta) \sin (\tilde{a}-\psi)+\sin (e) \cos (\theta)
$$

\section{Optimization Procedure}

Numerical approximations of optimal paths are obtained by discretizing the problem as follows. For a chosen number of knots $n \geq 1$, we subdivide the interval $\left[t_{o}, t_{f}\right]$ into $n$ subintervals $\left[t_{o}, t_{1}\right],\left[t_{1}, t_{2}\right], \ldots .,\left[t_{n-1}, t_{f}\right]$ of unequal duration. Let each time interval be defined $\Delta t_{j}=t_{j+1}-t_{j}$. In each subinterval, we assume the control input is constant, i.e. $(z(t))=\left(z_{j}(t)\right), t \in\left[t_{j}, t_{j+1}\right]$, where the parameters $\left(z_{j}(t), \Delta t_{j}(t)\right), 0 \leq j \leq$ $n-1$, are unknown.

We produce periodic splines by selecting knot points for $z_{j}(t)$, and $\Delta t_{j}(t), 0 \leq j \leq n-1$, where $n=21$ and using the MATLAB ${ }^{\circledR}$ functions csape and fnval. The spline for $\psi$ is taken as a constant, and computed from the optimal velocity and given cylinder radius, $\dot{\psi}=\frac{2 \pi V_{\text {Power }} \text { min }}{r}$. We differentiate these splines using the function fnder to obtain values for $\dot{\psi}(t), \ddot{\psi}(t), \dot{z}(t)$, and $\ddot{z}(t)$.

We treat the parameters $\left(z_{j}(t), \Delta t_{j}(t)\right), 0 \leq j \leq n-1$ as inputs to a nonlinear optimization problem. Constraints upon this problem are imposed from the boundary conditions (24)-(26). From (23), the objective function is the total energy at the end of flight. We then numerically solve for optimal flight paths using the MATLAB $^{\circledR}$ Optimization Toolbox function fmincon. The constraint tolerance was $10^{-2}$ while the function tolerance was $10^{-2}$.

\section{Example Aircraft Parameters}

\begin{tabular}{|c|c|c|}
\hline Parameter & Symbol & Value \\
\hline Solar cell efficiency & $\eta_{u}$ & 0.19 \\
\hline Propeller efficiency & $\eta_{p}$ & 0.70 \\
\hline Wing span & $b$ & $3.10 \mathrm{~m}$ \\
\hline Wing area & $S$ & $0.56 \mathrm{~m}^{2}$ \\
\hline Airplane weight & $W$ & $39.24 \mathrm{~N}$ \\
\hline Stall Constraint & $\alpha_{m a x}$ & $18.00^{\circ}$ \\
\hline Oswald efficiency factor & $\eta_{e}$ & 0.91 \\
\hline Solar spectral density & $P_{s d}$ & $886 \mathrm{~W} / \mathrm{m}^{2}$ \\
\hline Air Density & $\rho$ & $1.29 \mathrm{~kg} / \mathrm{m}^{3}$ \\
\hline
\end{tabular}

Table 2. YellowTail Aircraft Parameters 